\title{
Sarah Nagel
}

\section{Staatskapitalismus goes global}

Chinas Wirtschaft wächst seit mehr als dreißig Jahren rasant. Als aufholende Ökonomie und Exporteur profitiert China von einer besonderen Konstellation im globalen Kapitalismus, in der sich die Akkumulation der Industrieländer verlangsamt hat (ten Brink 2010: 35). In China hat sich währenddessen ein wettbewerbsorientierter Staatskapitalismus herausgebildet, in dem staatliche Akteure profitorientiert handeln. Doch die chinesische Wirtschaftspolitik steht an einem Wendepunkt, da sich die Wachstumsraten der vergangenen Jahre nicht unendlich fortsetzen lassen. Im dritten Quartal 2012 sank das Wachstum erstmals seit dem Beginn der Wirtschaftskrise 2008 unter die magische Grenze von 8 Prozent, die als notwendig gilt, um ausreichend Menschen in den Arbeitsmarkt integrieren zu können und die politische und soziale Stabilität zu wahren. Dabei ist China auch von der Konjunktur der Länder abhängig, in die chinesische Waren exportiert werden. 2008 wirkte sich die Krise in China aus, indem zwischen 11 und 20 Millionen Wanderarbeiter ihre Arbeit verloren (Schmalz 2010: 489). Südlich von China wächst gleichzeitig die Lohnkonkurrenz südostasiatischer Länder. Den wachsenden inneren und äußeren Problemen versucht die Regierung mit verschiedenen Maßnahmen gegenzusteuern. Eine der Strategien ist die Go Global-Politik, mit der die Regierung seit mehr als zehn Jahren versucht, chinesische Unternehmen international und auf dem heimischen Markt wettbewerbsfähig zu machen und neue Absatzmärkte zu erschließen. Die chinesischen Auslandsdirektinvestitionen sind seither drastisch angestiegen und die globale Verschiebung zeigt sich auch hier: Während der weltweite Trend der Auslandsdirektinvestitionen (ADI) seit Beginn der Wirtschaftskrise ab 2008 rückläufig ist, stiegen die chinesischen ADI in den Jahren 2008 und 2009 erheblich an (Brod/Schüler-Zhou/Schüller 2010: 1). Die Investitionen werden dabei überwiegend von staatlichen Unternehmen getätigt. Im Folgenden soll erläutert werden, wie die kapitalistische Entwicklung in China nicht nur mit einem wachsenden Privatsektor einherging, sondern auch mit einer Umstrukturierung und Neuorientierung von staatlichen Unternehmen. Während viele kleine und mittlere Staatsunternehmen privatisiert wurden, wurden große Unternehmen neu orientiert und in ihrer Tendenz zur Konzentration gefördert. Im ersten Teil werden daher die kapitalistische Entwicklung in China skizziert und die staatlich geförderte Konzentration beleuchtet. Im zweiten Teil 
wird die Go-Global-Politik näher erläutert und beispielhaft aufgezeigt, welche Interessen dahinter stehen und wie sie sich auswirkt.

\section{Kapitalistische Entwicklung in China}

Die Kommunistische Partei nennt China weiterhin eine „sozialistische Marktwirtschaft", doch das will ihr mittlerweile kaum noch jemand abnehmen. In vielen Publikationen ist mittlerweile von Staatskapitalismus die Rede. „Typische Kennzeichen des Kapitalismus wie der Zwang zur Akkumulation des Kapitals, eine end - und maßlose Wachstumsorientierung, Wettbewerbsprinzipien, zweckrationale Nützlichkeitserwägungen sowie ausgeprägte soziale Gegensätze haben die Ökonomie zu einem neuen Zentrum des globalen Kapitalismus gemacht“, schreibt Tobias ten Brink (ten Brink 2011a: 4). Er hat die Entwicklung des Kapitalismus in China analysiert und argumentiert, dass sich hier mit dem marktliberalen, wettbewerbsgetriebenen Staatskapitalismus eine eigene Spielart des Kapitalismus herausgebildet hat (ten Brink 2010: 9). Dessen Entwicklung lässt sich grob in zwei Phasen einteilen. Die erste Phase ab 1979 war geprägt durch kleinere, private Unternehmen, in der zweiten Phase ab 1992 begannen sich die großen, profitorientierten Staatsunternehmen zu entwickeln, die heute die chinesische Volkswirtschaft prägen und auch bei den Auslandsdirektinvestitionen eine tragende Rolle spielen.

Erste Phase: Begonnen hat die Geschichte des chinesischen Wachstums auf dem Land, nachdem die Regierung anfangs im Landwirtschaftssektor mit Reformen experimentiert hatte und Bauern zum Beispiel einen Teil ihrer Erzeugnisse zu nicht festgelegten Preisen verkaufen konnten. Als Resultat des ersten Experimentierens stiegen Produktivität und Verbrauch vor allem auf dem Land an. Gewinne wurden anschließend oft in kleine, private Unternehmen investiert. Haushalte außerhalb der Städte wurden damit quasi zu einem Katalysator für die Kapitalakkumulation (McNally 2006). In dieser ersten Phase waren private Firmen und Unternehmertum politisch kaum anerkannt, auch die Entscheidungsgewalt von Unternehmern war noch nicht ausgeprägt. Bis 1988 durften Firmen außerdem nur acht Mitarbeiter haben. Viele Unternehmer umgingen Einschränkungen daher dadurch, dass sie den sprichwörtlichen „roten Hut“ aufsetzten und ihre privaten Firmen als staatliche Unternehmen oder sogenannte collectively owned enterprises (COEs) registrierten, die von den Lokalregierungen kontrolliert wurden. Der Anteil dieser versteckten privaten Firmen zu dieser Zeit wird auf ein Drittel bis hin zu 90 Prozent geschätzt (ten Brink 2010: 10). Für diese Praxis benötigten Unternehmer, wenn sie nicht selbst Kader waren, gute Beziehungen zu Entscheidungsträgern in der Kommunistischen Partei. 
Zweite Phase: In der zweiten Reformphase ab 1992 begann die Regierung, eine Politik zugunsten von großen Staatskonzernen zu betreiben. Etliche kleinere Staatsbetriebe wurden geschlossen oder privatisiert. Gleichzeitig wuchs durch Unternehmensgründungen und Privatisierungen der private Sektor. Als Folge wurden Arbeiter massenhaft entlassen. Im Jahr 2000 hatten state owned enterprises (SOEs) und COEs schließlich noch etwa 96 Millionen Angestellte, während es in privaten und halbprivaten Unternehmen 72 Millionen waren. Die Zahl der Arbeitskräfte in kleinen Privatbetrieben und selbständigen Beschäftigungsverhältnissen war auf ca. 48 Millionen im Jahr 2000 rasant gestiegen (Lüthje 2006: 64). Auch die Zahl der Arbeitsmigranten aus ländlichen Gegenden hat stark zugenommen. 2000 lag sie bei etwa 150 Millionen, 2006 bereits bei 200 Millionen Arbeitern im sekundären und tertiären Sektor (ten Brink 2010: 24).

Unternehmertum wurde schrittweise anerkannt und Parteikader kauften plötzlich reihenweise staatliche Unternehmen und COEs durch sogenannte management buyouts auf. Wie das geschah, zeigt beispielhaft eine Brennerei, deren Weg durch die Reformen Anita Chan und Jonathan Unger untersucht haben. Im Zuge einer Privatisierungswelle ab den späten 1990er Jahren war 2003 die Brennerei an der Reihe. Zu diesem Zeitpunkt war der Ausdruck management buyout in China bereits so bekannt, dass in der Überschrift eines Zeitungsartikels über den Verkauf anstatt chinesischer Schriftzeichen die englische Abkürzung MBO stand. Offiziell wurde die Privatisierung EMBO genannt - employee management buyout. Die Arbeiter durften allerdings nur maximal 13 Prozent der Anteile kaufen. Den Großteil der Anteile kauften schließlich die 28 höchsten Manager der Brennerei, die sie zu ihrer staatlichen Zeit geführt hatten, für einen künstlich niedrig gehaltenen Preis (Chan/Unger 2009: 22). Mit der Zeit tauchten in der zweiten Reformphase immer mehr Unternehmer mit Parteibuch auf. Während 1991 nur 7 Prozent der Unternehmer angaben, Parteimitglied zu sein, waren es 2003 schon mehr als ein Drittel. Und nicht nur das: Je größer und umsatzstärker eine Firma war, desto wahrscheinlicher wurde sie von einem Parteimitglied geleitet (ten Brink 2011: 13). Die staatlich geförderte Konzentration der 1990er Jahre hält Huang Yasheng, der 2008 das Buch Capitalism with Chinese Characteristics veröffentlicht hat, aus marktliberaler Sicht für falsch - für ihn sind es die kleinen, privaten Firmen auf dem Land der 1980er Jahre, welche die kapitalistische Dynamik ausmachten. „China war erfolgreich, wenn privates Unternehmertum von unten florierte und es stagnierte, wenn Unternehmertum unterdrückt wurde“, erklärt er (Huang 2008: 7, Übers. v.d.A.). Staatliche Intervention hält er für hinderlich und beschuldigt die Regierung, durch ihre Eingriffe und eine Bevorzugung der Städte in den 1990er Jahren das wahre Unternehmertum ausgebremst zu haben. Andreas widerspricht ihm: „Market competition intrinsically centralizes property without the need for state interven- 
tion. Winners squeeze out losers, amass capital, capture market share and block new entrants." (Andreas 2010: 74).

\section{Staatlich geförderte Konzentration}

Allein zwischen 1996 und 1999 wurde die Zahl der Staatsunternehmen von 127.600 auf 61.300 reduziert. Nach dem Motto „fördern, was gefördert werden muss, und ausscheiden, was ausgeschieden werden muss" sollten kleinere Unternehmen abgestoßen und nur die 1.000 größten Staatsbetriebe weiterhin staatlich kontrolliert werden (Schmalz 2010: 488). Diese Entwicklung setzt sich auch heute noch fort: Zwar wächst der private Sektor in China kontinuierlich, aber große Staatskonzerne gewinnen ebenfalls an Kapital und Einfluss. Die 121 größten Staatskonzerne konnten 2002 noch 360 Mrd. US-Dollar auf sich vereinen, 2010 waren es bereits 2,9 Billionen (The Economist 2011). Börsennotierte SOEs spielen in der chinesischen Volkswirtschaft eine große Rolle und erhalten nach wie vor privilegierten Zugang zu staatlich kontrollierten Krediten, Subventionen und Land (ten Brink 2010: 20). 2003 wurde mit der SASAC (State Asset Supervision and Administration Commission) außerdem eine neue Aufsichts- und Verwaltungskommission für Staatsvermögen gegründet, welche die Staatsanteile und etwa 190 der größten nationalen Konzerne beaufsichtigt und die Unternehmen umstrukturieren kann, um sie profitabler zu machen. Etwa 500 besonders leistungsstarke Unternehmen werden außerdem als national champions betitelt und gefördert. Die SASAC war es auch, die nach ihrer Gründung die Diskussionen über den Verkauf eines Teils der Staatsanteile einleitete. Häufig wurden schließlich etwa 25 Prozent des Unternehmens an Einzelpersonen und institutionelle Investoren verkauft und an einem Börsenplatz notiert. Im Jahr 2006 existierten nach offiziellen Angaben der SASAC infolge von Reorganisation und Rationalisierung noch 161 ,zentrale SOEs“. Die Kommission kontrolliert Firmen in der Öl-, Metall-, Strom- und Rüstungsindustrie sowie den Bereich der Telekommunikation (ten Brink 2010: 20). SASAC hat die Aufgabe, staatliche Unternehmen für den globalen Markt umzustrukturieren und verdient seit 2007 auch daran, denn seither ist die Kommission anteilig an den Profiten der Unternehmen beteiligt. Die Firmen, die wenig rentabel sind, werden von der SASAC geschlossen oder müssen mit anderen Firmen fusionieren. Das Schließen und Verschmelzen führt einerseits dazu, dass Unternehmen den Profit an erste Stelle setzen müssen, wenn sie nicht abgeschafft werden wollen, und andererseits erhöht es die Wahrscheinlichkeit, dass für SASAC und die beteiligten Unternehmen Gewinne tendenziell wichtiger werden als strategische Interessen (Seifert 2008: 12ff). Die Kommission 
verfügt allerdings nicht über die Macht, Firmen unter ihre Aufsicht zu zwingen (Salidjanova 2011: 13).

Eines der wichtigsten Unternehmen unter SASAC-Verwaltung ist der Konzern Sinochem (China National Chemicals Import \& Export Corporation), der seinen Weg zu einem einflussreichen transnationalen Konzern lange vor der Go Global-Strategie begann. Sinochem wurde 1950 gegründet und hielt einst das Monopol auf den Import - und Export von Erdölprodukten und chemischem Dünger, bevor in den 1980er Jahren mehr Konkurrenz entstand und der Konzern sich entschloss, sich fortan internationaler und breiter gefächert aufzustellen und von einem chinesischen Außenhandelsunternehmen zu einem transnationalen Konzern umzuwandeln. Der Staatsrat bewilligte den Schritt 1987, und die Strategie funktionierte: Das Unternehmen entwickelte sich bis zum Jahr 2000 in einen Konzern mit über hundert Standorten und Joint Ventures im Ausland und wurde schon 1995 als eines der 500 größten Unternehmen weltweit aufgeführt (Hong/Sun 2006: 624). Sinochem bezeichnet sich heute als „das Schlüsselunternehmen unter SASAC-Verwaltung“, sieht sich als „marktorientiertes, globales Konglomerat" und verfügt mittlerweile über mehr als 200 Filialen in - und außerhalb Chinas. SASAC wiederum zeigt sich durch eine sehr gute Bewertung der Unternehmensperformance erkenntlich. Als staatliches Unternehmen im Energiesektor sieht Sinochem es als seine Pflicht an, für Chinas Energiesicherheit zu sorgen und verbindet Profitstreben mit einem ideologischen Überbau. Die Geschichte von Sinochem steht beispielhaft für andere SOEs, die im Rahmen der Konzentrationspolitik gefördert wurden und auch im Ausland aktiv sind.

Finanzielle Unterstützung erhalten die Firmen bei Go Global-Aktivitäten durch staatliche Banken, deren Kreditvergabe politisch gesteuert werden kann. Die „großen vier“ Banken Bank of China, China Construction Bank, Agricultural Bank of China und Commercial Bank of China dominieren das chinesische Bankensystem und sind ihrerseits umstrukturiert und für ausländische Investoren geöffnet worden. Dass die Zentralregierung trotzdem die Kontrolle behält, ist jedoch an der Art ihrer Kreditvergabe deutlich erkennbar (Gottwald 2011:291). Daneben sind auch Banken wie die China Development Bank und die Export-Import Bank (Exim Bank) relevant, die Kredite für Investitionen im Ausland und Bürgschaften vergibt und auch für einen Großteil der staatlichen Entwicklungshilfe in afrikanischen Ländern zuständig ist (Seifert 2008: 8). Da Finanzierung in China häufiger durch Bankkredite stattfindet als durch die Kapitalmärkte, ist es wesentlich, dass sich alle großen Banken in staatlicher Hand befinden: Staatliche Unternehmen haben dadurch einen weit leichteren Zugang zu Kapital als private. 


\section{Staatlicher vs. privater Sektor}

Während bei den Staatsunternehmen eine Tendenz zur Konzentration zu beobachten ist, ist gleichzeitig der private Sektor stark gewachsen. Die Größenverhältnisse zwischen staatlichem und privatem Sektor in China zu bestimmen ist nicht einfach, weil die Besitzerstruktur in den Mischformen der Firmen nicht immer gleich ist und sich seit Beginn der wirtschaftlichen Öffnung in vielen Fällen verändert hat. Als ,staatlich “ zählen klassische SOEs, während der nicht-staatliche Sektor neben vollständig privaten Firmen auch aus COEs, TVEs, Joint Ventures und Firmen mit mindestens 25 Prozent Auslandskapital (foreign invested enterprises, FIE) besteht. Während chinesische Statistiken den Anteil privater Firmen als sehr gering einschätzen, geht McNally von einem Anteil von mindestens 50 Prozent des Bruttoinlandsprodukts schon 1999 aus (McNally 2006: 24). Huang hat die Größenverhältnisse unter Beachtung der realen Kontrollrechte in den Unternehmen untersucht und kommt zu der vorsichtigeren Einschätzung, dass der Anteil privater Firmen an der gesamten industriellen Wertschöpfung 1998 28,9 Prozent betrug, 2001 44,7 und 2005 50,5 Prozent. Firmen mit einem Anteil chinesischen Kapitals machten dabei 1998 17,2, 2001 27,8 und 2005 19,1 Prozent aus (Huang 2008: 15). Die steigende Zahl setzt sich dabei aus Unternehmensgründungen und Privatisierungen von SOEs und COEs zusammen, die in den 1990er Jahren vorangetrieben wurde. Seit den 1990er Jahren konnte der Privatsektor so um jährlich durchschnittlich 35 Prozent wachsen (ten Brink 2010: 21). Staatliche und private Firmen treten dabei auch in Konkurrenz zueinander. Besonders seitdem China 2001 der WTO beigetreten ist, stehen Staatsunternehmen unter Druck, weil Wirtschaftsbereiche für Privatunternehmen und auch Joint Ventures weiter geöffnet werden mussten. Allerdings wissen sich staatliche Akteure in vielen Fällen zu helfen: Obwohl Joint Ventures nach dem Beitritt im Telekommunikationssektor aktiv werden durften, waren die finanziellen Anforderungen und bürokratischen Hürden so hoch, dass bis 2010 keine einzige Lizenz vergeben wurde. Der Bereich wird also weiterhin von Staatskonzernen dominiert. Im Luftverkehr haben die drei staatlichen Konzerne Air China, China Southern und China Eastern einen Preiskrieg begonnen, als ihnen nach 2001 acht private Firmen Konkurrenz machten. Die privaten Konkurrenten konnten weder das chinesische Reservierungssystem nutzen, noch erhielten sie Treibstoff, auf den der Staat ein Monopol hat, unter denselben großzügigen Konditionen wie die SOEs. Als diese wiederum infolge der Wirtschaftskrise und Managementfehlern in finanzielle Schwierigkeiten gerieten, griff ihnen der Staat unter die Arme. China Eastern erhielt eine Milliarde US-Dollar, China Southern 430 Millionen und Air China 220 Millionen (Wines 2010). Nicht nur private und staatliche Unternehmen stehen in Konkurrenz, sondern auch SOEs untereinander. Das 
wird dadurch verstärkt, dass auch die Regionen miteinander konkurrieren. Der Konzentrationskurs der Zentralregierung, die international wettbewerbsfähige national champions schaffen will, gerät dabei zeitweise in Widerspruch mit den Interessen lokaler Kader und Unternehmen. Ein Beispiel ist die Stahlindustrie, die von der Zentralregierung als zentrale Branche angesehen wird. Drei chinesische Stahlkonzerne befinden sich derzeit unter den weltweit größten, die restliche Stahlindustrie in China ist weit verstreut. Einer der drei Riesen, ist SASACUnternehmen Baosteel: Der Stahlkonzern Baosteel wurde 1978 als Baoshan Iron and Steel Corporation gegründet und hatte 32.000 Mitarbeiter. Heute ist Baosteel ein international tätiger Konzern mit 116.702 Angestellten. Im Zuge der regierungsverordneten Konzentrationsbemühungen sollte Baosteel weiter wachsen und prüfte die Übernahme von lokalen SOEs, stieß dabei aber auf Widerstand: Um sich gegen den Zusammenschluss zu wehren, wurden 2009 in der Provinz Hebei mehrere Unternehmen zusammengefasst und bilden nun unter dem Namen Hebei Iron and Steel selbst einen der weltgrößten Stahlkonzerne. Dieser wiederum kaufte Anteile an zwölf privaten lokalen Stahlfirmen auf(Kyle/ Szamosszegi 2011: 32). Insgesamt dominieren staatliche Unternehmen zwar einige Bereiche, müssen durch die Konkurrenz mit dem wachsenden Privatsektor und die Öffnung für internationale Firmen aber trotzdem auf ihre Wettbewerbsfähigkeit achten. Da gleichzeitig auch die Durchschnittslöhne steigen, gerät China insgesamt als „Werkbank der Welt“ unter Druck. Unternehmen müssen Fähigkeiten als Finalproduzenten entwickeln, um längerfristig wettbewerbsfähig zu bleiben. Das aber ist teuer und aufwendig. Eine Strategie, um Know-How und Technologie nach China zu bringen und dem Ziel einen Schritt näher zu kommen, sind Investitionen im Ausland.

\section{Die "Go Global"- Strategie - wie investieren chinesische Unternehmen im Ausland?}

Die Auslandsdirektinvestitionen aus China sind in den vergangenen Jahren stark angewachsen. Der Gesamtbestand der ADI betrug im Jahr 2000 noch $28 \mathrm{Mrd}$. US-Dollar und 2008 bereits $148 \mathrm{Mrd}$. (Schmalz 2010: 493). Damit bewegen sich die chinesischen ADI zwar auf einem im Vergleich mit Industriestaaten niedrigen Niveau, wachsen aber mit einer viel höheren Dynamik. Die Go Global-Strategie ist Teil des zehnten Fünfjahresplans. Mit der Strategie verfolgte die Regierung vor allem fünf Ziele: Eine breitere Streuung von Wirtschaftszweigen und Regionen bei Investitionen, effektivere Ausbeutung von Ressourcen, die Stärkung des Außenhandels, die Vorbereitung und Unterstützung von - insbesondere großen - Firmen bei ihren Auslandsaktivitäten, eine allgemeine Verstärkung und 
Diversifizierung von Auslandsaktivitäten und die Förderung von Firmenkäufen und Zusammenschlüssen durch chinesische Unternehmen (Gottwald 2011:285). Dabei stehen den Unternehmen eine Reihe von staatlichen Institutionen unterstützend zur Seite. Nach wie vor entscheidet die Regierung darüber, in welche Wirtschaftszweige und Märkte Investitionen fließen sollen (Salidjanova 2011: 4). Einige Ziele werden in einer gemeinsamen Veröffentlichung der einflussreichen Planungskommission (National Development and Reform Commission, NDRC) und der China Import-Export Bank aus dem Jahr 2004 deutlich, in der sie dazu aufrufen, Investitionen in folgenden Bereichen zu fördern: Erstens Projekte zur Erkundung von Rohstoffen, um den heimischen Mangel an natürlichen Ressourcen auszugleichen; zweitens Projekte, die den Export von chinesischen Technologien, Produkten, Ausstattung und Arbeitskräften fördern; drittens die Unterstützung von Projekten zur Forschung und Entwicklung im Ausland, um hochentwickelte Technologien, neue Management-Techniken und Fachleute besser nutzen zu können; und viertens Einkäufe und Zusammenschlüsse von Firmen, welche die Wettbewerbsfähigkeit chinesischer Firmen und deren Zugänge zu internationalen Märkten verbessern können. Im Jahr 2004 hat das Handelsministerium MOFCOM außerdem gemeinsam mit dem Außenministerium erstmals Investitionsrichtlinien für chinesische Unternehmen herausgegeben, um die Go Global-Politik in die von der Regierung gewünschte Richtung zu lenken. Der Investitionskatalog soll den Unternehmen bei der Wahl der Zielländer und Industrien den Weg weisen. Der Katalog umfasste Empfehlungen für 67 Länder und sieben Industrien, darunter 26 asiatische Länder (drei in Zentralasien), 13 afrikanische Länder, zwölf europäische Länder (zehn davon EU-Mitgliedsländer), elf Länder in Nord- und Südamerika und fünf in Ozeanien. In den folgenden Jahren wirkte auch die Planungskommission NDRC an den Katalogen mit (Brod/Schüler-Zhou/Schüller 2010: 4). Der Staatsrat stellte gleichzeitig Steuernachlässe, finanzielle Hilfen und weitere Maßnahmen zur Verfügung, wenn Firmen Auslandsaktivitäten planten (Salidjanova 2011: 5). „Da ein übergreifendes außenwirtschaftspolitisches Ziel in China die Bildung nationaler Flaggschiffunternehmen ist - unter 'nationalen Champions' werden protegierte Firmen verstanden, die auf der Basis starker Positionen auf dem Heimatmarkt suksessive einen transnationalen Einfluss geltend machen - werden die Bemühungen der Unternehmen politisch flankiert..." schreibt ten Brink (2011b: 13).

Die Strategie zeigte bald ihre Wirkung. Das OECD-Investmentkomitee gab 2008 bekannt, die chinesischen Auslandsdirektinvestitionen seien seit dem Jahr 2000 um das Neunzehnfache auf mehr als 18 Mrd. US-Dollar angewachsen. Rund zwei Drittel der ADI werden dabei von Staatskonzernen getätigt (Brod/ Schüler-Zhou/Schüller 2010: 6). Die bei chinesischen Firmen beliebteste Form der Investition sind dabei Fusionen und Übernahmen (mergers \& acquisitions, 
M\&A), weil sie kostengünstiger sind als eine eigenständige Marktplatzierung (Gong 2012: 7). Laut einer Studie aus dem Jahr 2011 ist die Anzahl der M\&AGeschäfte in den letzten Jahren um jährlich 106,8 Prozent gewachsen (Gong 2012: 8). Bei Investitionen in Europa besteht der M\&A-Trend erst seit 2007, davor haben chinesische Investoren eher ohne bestehende Strukturen investiert (Hay/Milelli 2011: 157). Mittlerweile sind mehr als 13.000 chinesische Firmen im Ausland präsent und investieren in mehr als 180 Ländern (Vendryes 2012: 5).

Regionale Verteilung: Mit 75 Prozent floss der weitaus größte Teil der Investitionen nach Asien inklusive den Mittleren Osten. 12 Prozent gingen nach Lateinamerika, je vier Prozent nach Afrika und Europa, 3 Prozent nach Ozeanien und 2 Prozent nach Nordamerika (Salidjanova 2011: 15). Diese Verteilung ist allerdings dadurch verzerrt, dass an der Spitze der Liste Steueroasen zu finden sind (z.B. im Jahr 2009 Hongkong, die British Virgin Islands und die Cayman Islands). Das lässt sich unter anderem dadurch erklären, dass einige chinesische Unternehmen ein sogenanntes round-tripping praktizieren, indem sie Geld in Steueroasen verschieben, um anschließen verdeckt auf dem chinesischen Festland zu investieren, da sie dort als ausländische Investoren Vorteile genießen. Werden die Steueroasen nicht mit eingerechnet, sieht die Verteilung etwas anders aus: Asien erhält dann noch 41,5 Prozent, Afrika 20,9 Prozent, Europa 13,8 Prozent, Ozeanien (insbesondere Australien) 10,2 Prozent, Nordamerika 9,8 Prozent und Lateinamerika 3,8 Prozent (Brod/Schüler-Zhou/Schüller 2010: 3). Langsam beginnen sich die Ziele der Investitionen jedoch zu ändern. Entwicklungs und Schwellenländer erhalten immer noch den größten Anteil der ADI, doch chinesische Banken wenden sich in letzter Zeit verstärkt auch Industriestaaten zu (Gong 2012: 8).

Sektorale Verteilung: Laut MOFCOM-Statistiken befanden sich 2009 17,3 Prozent des Gesamtbestands chinesischer ADI im Primärsektor, 5,5 Prozent im Sekundärsektor und 77,1 Prozent im tertiären Sektor. Im Primärsektor wird der überwiegende Teil der Investitionen im Ressourcenbereich getätigt, im sekundären Sektor geschehen alle Investitionen im Bereich Manufaktur und im tertiären Sektor teilt sich der Großteil der ADI unter Finanzdienstleistungen sowie Groß- und Einzelhandel auf. Die Verteilung auf Sektoren ist seit dem Jahr 2004 relativ konstant geblieben (Davies 2010: 9).

\section{Staatliche Akteure der Go-Global-Politik}

Chinesischen Unternehmen, die den Schritt ins Ausland planen, steht eine Reihe staatlicher Institutionen zur Seite. Hier soll ein Überblick über die wichtigsten gegeben werden. Auf einer übergeordneten Steuerungsebene legt der Staatsrat 
grundlegende Politiken und Maßgaben der Außen - und Entwicklungspolitik fest, während die Planungskommission (National Development and Reform Commission, NDRC) die Wirtschaft durch Rahmenvorgaben und Ressourcenverteilung zu steuern sucht. Die NDRC hat in den vergangenen fünfzehn Jahren an Bedeutung verloren, aber staatseigene Unternehmen sind nach wie vor durch Vorgabensysteme an die Kommission gekoppelt. Seit 2005 wird die NDRC durch die State Energy Leading Group (SELG) ergänzt, deren Hauptaufgabe es ist, die Energiesicherheit des Landes zu beobachten und bei Bedarf Maßnahmen zu ergreifen um diese zu erhalten, was vor allem für jene Betriebe wichtig ist, die hauptsächlich im Bereich natürlicher Ressourcen tätig sind (Seifert 2008:10). Das Handelsministerium (Ministry of Commerce, MOFCOM) beobachtet die Entwicklungstendenzen wirtschaftlicher Globalisierung und macht Vorschläge dazu; außerdem ist es für Gesetze und Regelungen zu auswärtigen Investitionen zuständig und wickelt Projekte der Entwicklungs - und Wirtschaftshilfe ab (Homepage des MOFCOM).

Für die Umsetzung der Investitionen selbst stehen der Regierung außerdem verschiedene Vehikel zur Verfügung. Die wichtigsten sind die State Administration of Foreign Exchange (SAFE) und die China Investment Corporation (CIC). Im Jahr 2003 gaben SAFE und MOFCOM außerdem den Weg dazu frei, dass ausländische Direktinvestitionen von weniger als drei Millionen US-Dollar auch auf der Provinzebene anstatt auf der zentralstaatlichen Ebene bewilligt werden durften. Was folgte, war „eine Buchstabensuppe aus Ämtern, Bürokraten und Unternehmen, die die Auslandsinvestitionen regulieren oder davon profitieren wollten." (Salidjanova 2011: 13). SAFE hat von der Zentralbank die Aufgabe übernommen, die ausländischen Devisen zu verwalten. Nachdem China Ende 2006 Devisen im Wert von 1.500 Mrd. US-Dollar angehäuft hatte, mehrten sich die Gerüchte, dass die chinesische Führung nach Wegen suche, diese effektiver und mit mehr Gewinn zu nutzen. Weil die Investmentmöglichkeiten von SAFE eingeschränkt waren, wurde nach langer Diskussion schließlich CIC gegründet, Chinas eigener Staatsfonds, der formell dem Finanzministerium untersteht. CIC wurde mit $200 \mathrm{Mrd}$. US-Dollar ausgestattet und so weit wie möglich wie eine unabhängige, profitorientierte Einheit aufgebaut, um Sorgen um den Regierungseinfluss bei Investitionen entgegenzuwirken. Der Plan ging allerdings nicht auf, denn der Fonds wurde in der öffentlichen Debatte in den USA und Europa mehr als einmal dafür kritisiert, angeblich Regierungsgelder für Investitionen in sensiblen Bereichen verwendet zu haben. Umgekehrt stand CIC auch in China in der Kritik, als der Fonds bei Investitionen in den USA und Europa Verluste machte (Gottwald 2011: 291). 


\section{China sucht Zugang zu Märkten, Ressourcen und Technologien}

Im Folgenden soll ein kurzer Überblick über zwei Unternehmenstypen gegeben werden: Einerseits diejenigen, die Zugang zu Rohmaterialien und natürlichen Ressourcen erlangen wollen, und diejenigen, die Zugang zu Technologie, KnowHow und etablierten Marken suchen. Dabei versuchen chinesische Unternehmen, die Krise zu nutzen: „Im Krisenjahr 2009 gab es eine Reihe von größeren M\&ATransaktionen chinesischer Unternehmen im Ausland. Sie machen deutlich, wie intensiv sich China mit Hilfe der Staatsunternehmen den Zugang zu ausländischen Rohstoffreserven sichern will. Gleichzeitig verstärkt sich die Tendenz zur Übernahme von technologieintensiven Unternehmen." (Brod/Schüler-Zhou/ Schüller: 3)

Natürliche Ressourcen: Die Sicherung von Energie und Ressourcen ist noch immer wichtiger Bestandteil der Investitionspolitik. Nennenswerte Aufkäufe in den letzten Jahren waren zum Beispiel die Übernahme von Swiss Addax Petroleum durch Sinopec für 7,56 Mrd. US-Dollar und der Kauf des australischen Kohlenminenbetreibers Felix Resources Ltd. für 2,9 Mrd. US-Dollar. Auch Chinas größter Ölkonzern Petrochina erwarb für umgerechnet 1,82 Mrd. US-Dollar etwa 60 Prozent der Anteile an zwei kanadischen Unternehmen von Athabasca Oil Sands. Im ersten Halbjahr 2010 waren der Ankauf des neunprozentigen Anteils von Synacrude durch Sinopec mit einem Volumen von 4,7 Mrd. US-Dollar und die Investition der China Investment Corporation (CIC) in PennWest-Energy von 1,2 Mrd. US-Dollar bemerkenswert (Brod/Schüler-Zhou/Schüller, 2012: 3). Den stärksten Boom erlebten Investitionen in natürliche Ressourcen in den zehn Jahren ab den späten 1990er Jahren, was eine logische Konsequenz aus dem anhaltenden Wirtschaftswachstum darstellte. Die größte Hürde hatte zuvor mangelndes Kapital in harter Währung dargestellt, da Investitionen in natürliche Ressourcen meist großen Umfang haben und einen Großteil des Firmenkapitals in Anspruch nehmen. Nachdem große Staatsunternehmen an den Börsen in Hongkong und New York notiert waren, nahmen die Investitionen zu (Hong/ Sun, 2006: 621). Auslandsaktivitäten im Ressourcenbereich werden von großen Unternehmen wie der China National Petroleum Corporation (CNPC), der China Petroleum and Chemical Corporation (Sinopec) und der China National Offshore Oil Corporation dominiert, die jeweils ihrerseits Unterfirmen betreiben und eine Reihe von Gemeinschaftsunternehmen eingegangen sind. Auch im Bereich Metall, Aluminium und Bergbau hat es eine ähnliche Entwicklung gegeben: die Großkonzerne China Nonferrous Metals Mining (CNMIM), Chinese Aluminium Group (CAG) und Minmetals (CMN) haben jeweils mehrere kleine Firmen geschluckt und werden von der SASAC unter Druck gesetzt (Seifert 2008: 12). Die Zentralregierung hatte über mehrere Jahre hinweg versucht, die fragmentierte Bergbaubranche in eine überschaubare Anzahl von Unternehmen 
umzuwandeln, die international eine signifikante Rolle spielen können. Wie ein hoher Regierungsbeamter sagte, sollten die staatlichen Bergbauunternehmen größer im Volumen und optimierter in ihrer Struktur werden sowie zu führenden Weltunternehmen wachsen und damit Einfluss auf die internationalen Mineralienpreise gewinnen (Seifert 2008).

Beispiele: Schlagzeilen machten chinesische Konzerne vor allem durch umfangreiche Investitionen in Afrika. Staatliche Firmen investierten in Afrikas natürliche Ressourcen, die Regierung baute ihre diplomatischen Beziehungen aus und gewährte Entwicklungshilfe, Steuernachlässe und zinsfreie Kredite, und auch private Firmen investierten in Afrika. Manche sprachen von einem „neuen Wettlauf um Afrika“ und Kritiker bemängelten, dass China auch in instabilen Regionen Geschäfte machte, aus denen westliche Regierungen und Firmen sich zurückgezogen hatten. Im Fokus der Kritik standen Investitionen im ressourcenreichen Sudan, der nicht nur für chinesische Firmen interessant ist: In den 1970er Jahren kauften sowohl die US-amerikanische Firma Chevron als auch die französische Total im großen Maßstab Konzessionen, um Öl fördern zu können. Ebenso waren die schwedische Lundin, die kanadische Talisman und österreichische Firmen im Sudan aktiv. Westliche Unternehmen verkauften jedoch ihre Konzessionen, nachdem sie durch Regierungen, NGOs und die Öffentlichkeit zunehmend unter Druck gerieten. Internationale und insbesondere kanadische Menschenrechtsorganisationen machten darauf aufmerksam, dass ein Zusammenhang zwischen Krieg und Vertreibung im Sudan bestehe, weil in Gebieten Konzessionen verkauft und Pipelines gebaut wurden, aus denen die Bevölkerung vertrieben worden war. 2005 waren fast alle Förderrechte an chinesische, indische und malaysische Unternehmen verkauft (Wagner 2005:2).

Tatsächlich sind die Investitionen aus China in Afrika von 2003 bis 2006 um das Siebenfache gestiegen und kaum ein anderes Schwellenland investierte so viel in Afrika wie China. Zudem flossen die Investitionen hauptsächlich in ressourcenreiche Länder wie Südafrika, Sambia, Algerien, Nigeria und den Sudan (OECD-Investmentkomitee 2008:2). Skepsis erregten besonders die Ölgeschäfte chinesischer Staatsunternehmen und Geschäfte nach dem sogenannten „AngolaModell“, deren Namensgeber ein Vertrag zwischen Angola und China über einen Kredit von einer Milliarde US-Dollar für die Wiederherstellung der Infrastruktur mitsamt Kraftwerksbau, Telekommunikation, Straßen und Eisenbahnen ist, der über die Zusicherung der Lieferung von 10000 Barrel Öl am Tag abbezahlt wird. Nach dem Angola-Modell werden Kreditlinien abgewickelt, indem der chinesischen Regierung ein Projekt unterbreitet und dann festgelegt wird, in welcher Form ein Kredit zustande kommt. Das profitierende Land wählt anschließend die Unternehmen aus, die den Auftrag erfüllen sollen und bietet im Gegenzug eine Lizenz zum Abbau bestimmter Bodenschätze an, über welche die Rückzah- 
lung des Kredits erfolgt. Als Abrechnungsstelle dient die Exim-Bank, welche die chinesischen und einheimischen Firmen bei Vertragserfüllung bezahlt. Die Rückzahlung erfolgt dann wiederum durch die vereinbarte Menge von Rohstoffen, die häufig ebenfalls ein chinesisches Unternehmen im Land fördert. Das Projekt wird währenddessen sowohl durch das Land überwacht, in dem es errichtet wird, als auch durch die Wirtschaftsbüros in den chinesischen Botschaften vor Ort. Das Projekt geht schließlich in das Eigentum des Landes über, in dem es errichtet wurde. Bekannte Verträge wurden vor allem über Öl, aber auch Kakao, Eisenerze und Bauxit abgeschlossen (Seifert 2008: 15). Allerdings sind Chinas Investitionen in Afrika zwar rasant gewachsen, bewegen sich aber immer noch auf niedrigem Niveau: Unter allen Investitionen in afrikanischen Ländern zusammen machen sie laut OECD nur 1 Prozent aus (OECD-Investmentkomitee 2008: 2), auch wenn es in einigen Ländern ein größerer Prozentsatz ist. Chinesische Forscher betonten indessen auf einer Konferenz zum zehnjährigen Bestehen der Go GlobalStrategie im August 2011, dass sie die Kritik aus westlichen Ländern für nicht gerechtfertigt halten: Der Drang chinesischer Unternehmen sei wirtschaftlich bedingt, nicht politisch; chinesische Firmen würden mit interessierten Ländern Geschäfte machen und so eine Win-win Situation für beide Seiten schaffen. Die Strategie sei dabei ein Schritt, der ganz einfach aus der wirtschaftlichen Entwicklung Chinas entstehe. Zuerst hätten chinesische Firmen im Ausland ihre Produkte verkauft und sich im nächsten Schritt durch Kooperationsverträge - zum Beispiel für Infrastrukturprojekte - auf diesen Märkten positioniert, erklärt Lin Hongyu. Die letzte Phase des „Go Global“ würde schließlich Direktinvestitionen bedeuten. In den Augen der Kongressteilnehmer sah es so aus, dass westliche Länder mit zweierlei Maß messen würden: sie selbst hätten die Internationalisierung ihrer Firmen bereits vollzogen, und auch andere asiatische Länder wie Japan und Südkorea seien diesem Beispiel bereits gefolgt. „Als die westlichen Länder selbst im Ausland aktiv wurden“, meint Professor Jin Canrong „haben sie ihre Kanonenboote mitgebracht" (Vendryes 2012:5 -6).

Zugang zu Technologie und Markennamen: Auf den ausländischen Rohstoffsektor entfallen zwar nach wie vor hohe Anteile chinesischer Auslandsinvestitionen, chinesische Firmen verfolgen aber inzwischen auch verstärkt den Kauf von Technologien, den Aufbau von Forschung und Entwicklung (F\&E) und die Übernahme von bekannten Markennamen und Vertriebsnetzwerken. Dazu gehören unter anderem auch Aufkäufe von bekannten Marken in der Automobilbranche. (Brod/Schüler-Zhou/Schüller, 2010).Zu den Firmen, die auch in technologisch fortgeschrittenen Bereichen jetzt oder in naher Zukunft international wettbewerbsfähig sind, zählen zum Beispiel Lenovo in der Computerindustrie, Haier auf dem Haushaltselektronikmarkt, Huawei in der Kommunikationsausrüstung und die Firma TCL in der Herstellung von Fernsehgeräten (ten Brink 2011a: 12). 
Auch im Bereich der erneuerbaren Energien können chinesische Firmen bereits mit westlichen Technologieführern konkurrieren. Die chinesische Regierung fördert sie dabei und hat ihre Bereitschaft erklärt, das weiterhin zu tun, vor allem durch Kredite. Der Solarmodulhersteller Yingli Green Energy hat zum Beispiel mittlerweile einen Weltmarktanteil von acht Prozent und exportiert 45 Prozent seiner Module nach Deutschland und weitere 25 Prozent in andere europäische Länder. (Brod/Schüler-Zhou/Schüller, 2010: 4). Einkäufe, um Zugang zu Technologien zu erhalten, machen einen kleineren Teil der Investitionen aus, sind aber eine häufig gewählte Methode in entwickelten Ländern: „Chinesische Firmen suchen typischerweise nach Schnäppchen auf dem europäischen oder US-amerikanischen Markt - Firmen, die einen bekannten Namen haben, aber in finanzieller Notlage sind - , um auf diesen Märkten einen Fuß in die Tür zu bekommen und Fähigkeiten im Marketing zu lernen“, meint Salidjanova. In China sei ausreichend Kapital vorhanden, um in jedes beliebige Unternehmen $\mathrm{zu}$ investieren, wenn jemand es als für das nationale Interesse notwendig empfindet. China muss keine Jahrzehnte damit verbringen, Marken zu etablieren, sondern kann mithilfe von staatlich finanzierten Firmen einfach vorhandene Markennamen aufkaufen (Salidjanova 2011: 8).

Beispiele: Der Elektronikhersteller TCL, größter Fernseherhersteller Chinas, begab sich ab 2000 auf die Suche nach Möglichkeiten, international einflussreicher zu werden. Der Konzern kaufte, um auf diese Art in den europäischen Markt einzusteigen und einen international bekannten Namen zu nutzen, 2002 die deutsche Firma Schneider auf und gründete 2004 eine Joint Venture mit dem französischen Elektronikbetrieb Thomson. Thomson und TCL formten damit den größten Fernsehproduzenten der Welt. Wenig später formte TCL auch eine Joint Venture mit der ebenfalls französischen Alcatel. Der Deal mit Alcatel zahlte sich aus: TCL profitierte von Technologietransfer und dem etablierten globalen Netzwerk Alcatels, während sich Alcatel stärker auf Telekommunikationsinfrastruktur konzentrieren konnte (Hong und Sun 2006: 626 - 627). TCL möchte sich weiter internationalisieren: „Durch Fusionen und Übernahmen im Ausland sind wir nach der historischen Wandlung von einem chinesischen Unternehmen zu einem multinationalen Konzern den nächsten Schritt gegangen. In den letzten Jahren wurden unsere Aktivitäten als Reaktionen auf die Herausforderung der Globalisierung angesehen, aber nun wollen wir unsere nächsten Schritte im Ausland bewusst planen." (Homepage der TCL Group).

Auch chinesische Automobilhersteller haben in den letzten Jahren mehrmals in Industriestaaten investiert. Der private Konzern Geely hat 2010 z.B. den schwedischen Hersteller Volvo übernommen und die schwedisch-chinesische Gruppe die Firma Saab (Lörchner 2012). Durch Übernahmen und Joint Ventures können chinesische Unternehmen von Technologie und Design profitieren, 
während sich ausländischen Partnern der chinesische Markt öffnet. Teils äußert sich das auch darin, dass chinesische Autos beinahe identisch mit ausländischen Modellen sind, wie zum Beispiel der Mercedes C und der Geely Merrie 300. Der größte chinesische Autohersteller SAIC (Shanghai Automotive Industry Corporation) hält zwei Mrd. US-Dollar an Auslandsvermögen und nutzt internationale Geschäftspartnerschaften und Aufkäufe, um seine Produktpalette zu erweitern und Marktanteile zu gewinnen (Davies 2010: 3). SAIC ist schon früh Joint Ventures mit Volkswagen und General Motors eingegangen und verkauft Autos unter verschiedenen Firmennamen, die teils aus ausländischen Aufkäufen stammen. Eine der Marken ist die 2006 gegründete Roewe, deren Wagen auf alten Modellen der britischen Marke MG Rover basieren. SAIC hatte 2005 nach der Pleite MG Rovers Rechte gekauft, um deren Technologie nutzen zu können. Die älteste, aber wesentlich kleinere chinesische Firma Nanjing Auto kaufte indessen die Marke MG Rover. Später fusionierten SAIC und Nanjing Auto im Zuge von Regierungsplänen zur Umstrukturierung der chinesischen Autoproduktion (Economist 2008). 2011 formte SAIC außerdem eine Joint Venture mit der Bussparte von Volvo, um in China Busse zu produzieren. Die komplette Marke Volvo übernahm indessen Geely für 1,8 Mrd. US-Dollar von Ford. Chinesische Autohersteller bemühen sich, konkurrenzfähig zu werden und besitzen mittlerweile auch die Schlüsseltechnologie für die Herstellung von Elektrofahrzeugen (Brod/Schüler-Zhou/Schüller 2010: 3).

\section{Fazit}

Im Wettbewerb mit internationalen Unternehmen sind chinesische Unternehmen insbesondere seit der Go-Global-Strategie zunehmend im Ausland aktiv und werden dabei von zahlreichen staatlichen Institutionen unterstützt. Im Vordergrund der Investitionspolitik stehen bisher die Konsequenzen aus Chinas Stellung als aufholender Ökonomie, die versucht, ihre Defizite gegenüber weiter entwickelten Staaten auszugleichen. Dabei ist eine Weiterentwicklung erkennbar. Während sich Investitionen lange Zeit lediglich auf die Erschließung von Rohstoffzugängen und Liefernetzwerken beschränkten, begannen chinesische Firmen Mitte 2009 vermehrt die Chance zu nutzen, angeschlagene technologieintensive Markenfirmen zu erwerben (Schmalz 2010: 493). Die Wirtschaftskrise ab 2008 hat einen bestehenden Trend verstärkt: Während die Weltwirtschaft insgesamt zwischen 1980 und 2000 mit BIP-Wachstumsraten um 3,3 Prozent wuchs, waren es bei China im gleichen Zeitraum Raten von fast 10 Prozent. Dies änderte sich auch nicht in der Phase bis 2007, in der das weltweite Wachstum bei 3,2 Prozent lag und China mit 10,2 Prozent wuchs (Schmalz 2010: 493). 
Chinesische ADI zeigen einige besondere Merkmale: Rund Zwei Drittel der ADI stammen von Staatsunternehmen und staatliche Institutionen nehmen durch Investitionskataloge stärkeren Einfluss auf deren Investitionsziele, als es Maßgaben in westlichen Ländern tun würden (Brod/Schüler-Zhou/Schüller 2010: 4). Dabei geht eine Konzentration von Kapital und Einfluss innerhalb Chinas mit einer konzentrationsfördernden Wirkung außerhalb Hand in Hand. Insbesondere seit 1992 fördert die Regierung innerhalb Chinas die Entstehung großer, leistungsstarker Staatskonzerne, damit diese Chinas Interessen außerhalb - wie etwa die Sicherung von natürlichen Ressourcen - möglichst gut umsetzen können. Dies geschieht in erster Linie durch die Kreditvergabe staatlicher Banken und politische Vorgaben. In kapital - und technologieintensiven Branchen wie denjenigen der natürlichen Ressourcen oder der Automobilindustrie bedeutet die Größe einen Vorteil - so gibt es beispielsweise nur fünf chinesische Autohersteller, die in der Lage sind, mit großen ausländischen Firmen der Automobilindustrie effektiv zu kooperieren (The Economist 2008). Ob die Konzentrations- und Investitionspolitik aber erfolgreich bleibt, ist ungewiss. Zwar haben sich staatliche Konzerne schon in der Vergangenheit als ein Weg erwiesen, um Entwicklungsländer voran zu bringen. Ob und wie lange sich das in Chinas Fall weiter fortsetzen lässt, hängt auch von der weltwirtschaftlichen Lage und der politischen Situation innerhalb Chinas ab. Zudem hat ihre Größe einigen SOEs bisher Vorteile beschert. Ob sie sich aber langfristig als beweglich genug erweisen werden um erfolgreich zu bleiben, bleibt abzuwarten.

\section{Literatur}

Andreas, Joel (2010): A Shanghai Model? On Capitalism with Chinese Characteristics, in: New Left Review, Vol. 65: 63-85.

Brod, Magnus; Schüler-Zhou, Yun; Schüller, Margot (2010): Chinas Going Global - Finanzmarktkrise bietet Chancen fürchinesische Investoren im Ausland. GIGA Focus Nr. 8, Hamburg.

Chan, Anita; Unger, Jonathan (2009): A Chinese Enterprise under the Reforms: What Model of Capitalism? in: The China Journal, Nr. 62: 1-26.

Davies, Ken (2010): Outward FDI from China and itspolicy context. Columbia FDI Profiles.

Gong, Cheng: Mapping and sequencing the "Go Global" strategy, in: Facing the Risks of the "Go Global Strategy": 7-11, http://www.ecfr.eu/content/entry/ china_analysis_facing_the_risks_of_the_going_out_strategy

Gottwald, Jörn-Carsten (2011): Cadre-Capitalism Goes Global: Financial Market Reforms and the New Role fot the People's Republic of China in World Markets, in: Louis Brennan (Hg.): The Emergence of Southern Multinationals and their Impact on Europe. Basingstoke: 283-300.

Hay, Francoise; Milelli, Christian (2011): Chinese and Indian Firms in Europe: Main Characteristics and Presumed Impacts, in: Louis Brennan (Hg.): The Emergence of Southern Multinationals and their Impact on Europe. Basingstoke: 151-163.

Hong, Eunsuk; Sun, Laixiang (2006): Dynamics of Internationalization and Outward Investment: Chinese Corporations'Strategies, in: The China Quarterly, Nr. 187, 610 - 634. 
Huang, Yasheng (2008): Capitalism with Chinese Characteristics, Cambridge October 26, 2011. Kyle, Cole; Szamosszegi, Andrew (2011): Analysis of State - owned Enterprises and State Capitalism in China. U.S.-China Economic \& Security Review Commission Report, http://www.uscc. gov/researchpapers/2011/10_26_11_CapitalTradeSOEStudy.pdf

Le Van, Jade (2012): A road accident: the inside story of the Polish highway that wasn't built by Chinese firms, in: China Analysis: Facing the Risks of the "Going out Strategy": 3-5, http:// www.ecfr.eu/content/entry/china_analysis_facing_the_risks_of_the_going_out_strategy

Lörchner, Jasmin (2012): Die Chinesen erobern Europas Automarkt, Financial Times Deutschland Online, 13.6.2012. http://www.ftd.de/unternehmen/industrie/:saab-volvo-rover-chinesenerobern-europas-automarkt/70049958.html

McNally, Christopher (2006): Insinuations on Chinese Capitalism, East-West Center Working Paper No. 15, http://www.eastwestcenter.org/sites/default/files/private/PSwp015.pdf

McNally, Christopher A./Guo, Hong/Hu Guangwei (2007): Entrepreneurship and Political Guanxi Networks in China's Private Sector. East-West Center Working Papers, Nr. 19. Honolulu: East-West Center.

MOFCOMStatisticalBulletin 2010, http://hzs.mofcom.gov.cn/accessory/201109/1316069658609. pdf

New masters of the universe - How state enterprise is spreading. The Economist Online, 21. Januar 2012: http://www.economist.com/node/21542925

Salidjanova, Nargiza (2011): Going Out - An Overview of China's Outward Foreign Direct Investment. U.S.-China Economic \& Security Review Commission Staff Report, 30. März 2011. http://www.uscc.gov/researchpapers/2011/GoingOut.pdf

Schmalz, Stefan (2010): Chinas neue Rolle im globalen Kapitalismus, in: PROKLA 161: 483-503.

Seifert, Andreas (2008): China in Afrika. IMI-Studie 9/2008. http://www.imi-online.de/download/AS-China-Studie.pdf

OECD Investment News Nr. 6, März 2008: China’s Outward Foreign Direct Investment. http:// www.oecd.org/investment/investmentpolicy/40283257.pdf

Ten Brink, Tobias (2008): Geopolitik. Geschichte und Gegenwartkapitalistischer Staatenkonkurrenz, Münster 2008.

- (2010): Strukturmerkmale des chinesischen Kapitalismus, MPIfG Discussion Paper 10/1, http:// www.mpifg.de/pu/mpifg_dp/dp10-1.pdf

- (2011a): Kooperation oder Konfrontation? Der Aufstieg Chinas in der globalen politischen Ökonomie. MPIfG Working Paper 11/7, http://www.mpifg.de/pu/workpap/wp11-7.pdf

- (2011b): Institutional Change in Market-Liberal State Capitalism: An Integrative Perspective on the Development of the Private Business Sector in China, MPIfG Discussion Paper 2/11, Max-Planck-Institut für Gesellschaftsforschung, Köln.

The Home Team - Indigenous carmakers are working their way up. The Economist Online, 13. November 2008, http://www.economist.com/node/12544893

Vendryes, Thomas (2012): The Going out strategy: economic moves with political consequences, in: China Analysis: Facing the Risks of the „Go Global Strategy", 5-7, http://www.ecfr.eu/content/ entry/china_analysis_facing_the_risks_of_the_going_out_strategy

Wagner, Jürgen (2005): Der Sudan als Spielfeld der Mächte. IMI-Analyse 2005/012 - in: Das andere Afrika: Widerstand gegen Krieg, Korruption und Unterdrückung, Hrsg. von Connection e.V. und der Antimilitaristischen Angolanischen Menschenrechtsinitiative e.V. (IAADH)

Wines, Michael (2010): China Fortifies State Businesses to Fuel Growth. The New York Times Online, 29.8.2010, http://www.nytimes.com/2010/08/30/world/asia/30china. html? pagewanted=all\&_r $=0$ 